\title{
Hyperbranched polyglycerol as an additive for water-based printing ink
}

\author{
Zuzanna Żołek-Tryznowska, Mariusz Tryznowski, \\ Justyna Królikowska
}

(C) The Author(s) 2015. This article is published with open access at Springerlink.com

\begin{abstract}
In this work, the application of hyperbranched polyglycerol as a performance additive for water-based printing inks is demonstrated. The hyperbranched polyglycerol, (BPG), was synthesized through anionic ring-opening polymerization from glycidol using 1-butanol as the starting material. The branched structure of BPG is presented and described. The presence of linear, dendritic and terminal units was confirmed by Moore et al. (Prog Surf Sci 88(3):213-236, 2013) ${ }^{13} \mathrm{C}$ NMR spectroscopy. BPG was then used as a performance additive in a water-based flexographic printing ink. The impact of a small amount of hyperbranched BPG on the printing ink color was examined by studying the optical density of a full-tone area, the total color difference, $\Delta E_{a b}{ }^{*}$, and the gloss of the dried ink film. The colorfastness to dry and wet rubbing of overprinted samples was studied. The investigated BPG is shown to be able to improve colorfastness to dry and wet rubbing of prints.
\end{abstract}

Keywords Hyperbranched polyglycerol, Print quality, Performance additives, Colorfastness

\section{Introduction}

The flexographic printing technology is suitable for printing on paper materials (coated and uncoated) and

Z. Żołek-Tryznowska ( $₫)$, J. Królikowska

Department of Printing Technology, Mechanics and Printing Institute, Faculty of Production Engineering, Warsaw University of Technology, Konwiktorska 2, 00-217 Warsaw, Poland

e-mail: z.tryznowska@wip.pw.edu.pl

M. Tryznowski

Faculty of Chemistry, Warsaw University of Technology, Noakowskiego 3, 00-664 Warsaw, Poland nonporous substrates, including metallized plastic films used in the packaging industry, using low-viscosity and rapid-drying fluids. ${ }^{1}$ There are three types of flexographic inks used for these applications, that is, waterbased, solvent-based and UV-curable ink. The attention in the printing industry is focused on the production of water-based printing inks in order to minimize the evaporation of organic solvents into the environment. The advantages of water-based inks are that water costs almost nothing and water does not pollute the environment. Printing with water-based flexographic inks has certain problems, as the adhesion of a water-based ink to the plastic film is worse and the resistance to dry and wet rubbing of overprinted plastic films is usually lower than that with solvent-based inks. ${ }^{2}$ Another problem is the high surface tension of water $\left(72 \mathrm{mN} \mathrm{m}^{-1}\right)$ in contrast with organic solvents (about $20 \mathrm{mN} \mathrm{m}^{-1}$ ). This means that water does not wet the printable base, especially the plastic film. It is well known that the surface tension of a flexographic printing ink has to be lower than the surface free energy of the plastic film to allow proper wetting and adhesion between the layers of the ink film and the plastic film. Typically, the surface free energy of polyethylene and polypropylene film is lower than $32 \mathrm{mN} \mathrm{m}^{-1}$. Therefore, it is necessary to add a wetting agent or organic co-solvent into water-based inks to decrease the surface tension and to achieve proper wetting and print adhesion.

Hyperbranched polymers are a new class of polymers. They are globular, highly branched macromolecules with a large number of functional groups. Hyperbranched polymers exhibit polydispersity and irregularity in terms of branching and structure, ${ }^{3}$ and they can usually be synthesized using a one-step procedure with a high yield. Hyperbranched polymers exhibit unique chemical and physical properties. ${ }^{4-8}$ The properties of hyperbranched polymers are often affected by the structure and architecture of the macromolecule, in terms of backbone and functional 
end-groups, degree of branching, molecular-weight distribution and so forth. Nowadays, the most popular hyperbranched polymers are hyperbranched polymers terminated with hydroxyl groups, such as polyethers (based on glycidol, i.e., polyglycerol) and polyesters (based on 2,2-bis(hydroxymethyl)propionic acid, i.e., Boltorn $\left.^{\mathrm{TM}}\right) .{ }^{9}$ However, hyperbranched polyglycerols are the more promising group of hyperbranched polymers, because polyglycerols are biocompatible, environmentally friendly, biodegradable and nontoxic. ${ }^{10,11}$ Oligoglycerols (2-10 U) have been FDA approved; ${ }^{12}$ therefore, hyperbranched polyglycerols are suitable compounds for biomedical applications. ${ }^{13,14}$ Moreover, hyperbranched polyglycerols have already found applications as surfactants in liquids for treating lithographic printing plates ${ }^{15}$ and as organic solvents in aqueous inkjet-printing inks to prevent paper deformation. ${ }^{16}$

In previous work, it has been shown that commercially available hyperbranched polyesters, such as polymers from the Boltorn ${ }^{\mathrm{TM}}$ family, can improve colorfastness to rubbing of solvent-based printing inks. ${ }^{17,18}$ Polyglycerols, unlike polyesters, are much more stable against acidic or basic hydrolysis ${ }^{19}$; thus, they can be used as performance additives in waterbased printing inks. The advantage of polyglycerols compared to polyesters is the remarkable solubility in water, owing to the functional hydroxyl groups.

Hyperbranched oligoglycerols and polyglycerols can be obtained from glycidol through polymerization, according to an anionic ${ }^{12,20,21}$ or a cationic ${ }^{22}$ mechanism. Polymers obtained through anionic polymerization of glycidol exhibit a relatively controlled and moderately narrow molecular-weight distribution with a controlled degree of branching. However, cationic polymerization of glycidol leads to polyglycerols with relatively low molecular weights as well as more irregular structures and branching.

The purpose of this work was to present a new application for hyperbranched polyethers. In this work, a hyperbranched polyglycerol (BPG), was used to modify the properties of a water-based printing ink, including the color properties and the fastness of the overprinted ink film. The impact of BPG on several print-quality parameters (optical density, color values, gloss and mechanical resistance properties) was estimated.

\section{Experimental}

\section{Materials}

All of the reagents for BPG synthesis were purchased from Sigma-Aldrich (Poznan, Poland) and were used as received, without further purification.

The water-based printing-ink sample (Process) was supplied by Michael Huber (Poland). This printing ink is recommended for printing on plastic films. For printing, oriented polypropylene (oPP) and polyethylene terephthalate (PET) films were used. The plastic films were activated by a corona treatment, they were transparent and had a thickness of $20 \mu \mathrm{m}$ for oPP and $12 \mu \mathrm{m}$ for PET.

\section{Characterization of BPG}

The chemical structure of BPG was characterized on a Biorad FTS 165 FTIR spectrometer using $\mathrm{KBr}$ pellets. ${ }^{1} \mathrm{H}$ and ${ }^{13} \mathrm{C}$ NMR spectra were recorded at room temperature, using tetramethylsilane as an internal standard and deuterated solvent (DMSO- $d_{6}$ ), on a Varian VXR $400 \mathrm{MHz}$ spectrometer; the spectra were analyzed using MestReNovav.6,2,0-7238 (Mestrelab Research S.L) software. MALDI-TOF mass spectra were measured on a Bruker UltraFlex spectrometer using DHB (2,5-dihydroxybenzoic acid) as a matrix and Bruker Peptide Calibration Standard (1047.19$3149.57 \mathrm{Da})$ as a calibrant; the spectra were analyzed with flexAnalysis v.3.3 (Bruker Daltonik $\mathrm{GmbH}$ ) and Polymerix v.2.0 (Sierra Analytics) software.

\section{Polymer synthesis}

BPG was prepared through a ring-opening polymerization reaction, similar to previously reported methods. $^{20,23}$ Potassium $(0.4 \mathrm{~g})$ was dissolved in 1-butanol $(10.0 \mathrm{~g})$ at room temperature in a $500 \mathrm{~mL}$ flask equipped with a magnetic stirrer and a thermometer. Then, glycidol $(50.0 \mathrm{~g})$ was added drop-wise at a rate of $1 \mathrm{~mL} \mathrm{~h}^{-1}$ to the mixture using a dosing pump. The mixture was stirred for $7 \mathrm{~h}$ at $80^{\circ} \mathrm{C}$. Next, the product was dissolved in methanol and neutralized by filtering through a cation-exchange resin. Methanol was distilled off at $40^{\circ} \mathrm{C}$ under reduced pressure $(0.5 \mathrm{mmHg})$. The obtained product was a colorless, viscous liquid.

FTIR (KBr): $3374(-\mathrm{OH}) ; 2930,2872\left(-\mathrm{CH}_{2}\right) ; 1117$ $(\mathrm{C}-\mathrm{O}) ; 669(\mathrm{C}-\mathrm{H}) \mathrm{cm}^{-1}$.

${ }^{1} \mathrm{H}$ NMR $\left(400 \mathrm{MHz}\right.$, DMSO-d $\left.d_{6}, \Delta\right): 0.86(\mathrm{t}, 3 \mathrm{H}$, $\left.\mathrm{CH}_{3}\right), 1.24-1.36\left(\mathrm{~m}, 2 \mathrm{H}, \mathrm{CH}_{2}\right), 1.38-1.49\left(\mathrm{~m}, 2 \mathrm{H}, \mathrm{CH}_{2}\right)$, 3.2-3.7 (m, polyglycerol protons), $4.4-4.8(1 \mathrm{H}, \mathrm{OH})$ ppm.

\section{Ink preparation and printing}

The modified ink was prepared by drop-wise addition of a calculated weight of BPG to pure Process ink, while continuously stirring. The mass fraction of BPG was 0.01 , because the best print quality was obtained after the addition of 0.01 of the hyperbranched polyester. ${ }^{17,18}$ Then, the ink was stirred for $30 \mathrm{~min}$ using a mechanical stirrer (Silverson, UK). The rheological characteristics of the printing inks (the original Process printing ink and the ink with added BPG) were specified by the flow 
time in a flow cup (volume $100 \mathrm{~mL}$, outlet diameter $4 \mathrm{~mm}$ ), according to an ISO standard. ${ }^{24}$ The measurements were performed at $23^{\circ} \mathrm{C}$; the relative error in the measurements was less than $3 \%$ and the kinematic viscosity was $19 \pm 0.5 \mathrm{~s}$ for the printing inks.

Laboratory printing was carried out with a Flexiproof instrument (TMI Machines, UK). The printing speed was $60 \mathrm{~m} \mathrm{~min}^{-1}$ and the printing engagement was 71 and 48 for oPP and PET plastic films, respectively. The printing engagement is related to the difference in the printing pressure between the substrate (printing base) and the printing plate, and it depends on the film thickness; when the film thickness increases, the engagement pressure decreases. The printing plate, made of a photopolymer prepared by the digital laser-photochemical method, had dimensions of $260 \times 90 \mathrm{~mm}$ and a thickness of $1.7 \mathrm{~mm}$. The pressure between the anilox engraved roller $\left(6 \mathrm{~cm}^{3} \mathrm{~m}^{-2}\right.$ volume, resolution or line ruling of 160 lines $\mathrm{cm}^{-1}$ ) and the plate cylinder was 98. All factors were kept constant during the printing process (printing speed, anilox roller and printing pressure). Printing was performed under controlled environmental conditions of $23^{\circ} \mathrm{C}$ and $50 \%$ relative humidity $(\mathrm{RH}) .{ }^{25}$

\section{Characterization of prints}

The quality test, which measured the adhesion of the dried ink film to the board, was performed by a simple tape test. The test tape was firmly attached to the print and rapidly peeled off by hand. According to the ISO standard, ${ }^{26}$ the adhesion should be quantified at least $16 \mathrm{~h}$ after conditioning at $23^{\circ} \mathrm{C}$ and $50 \% \mathrm{RH}$. One test was carried out on two different overprinted strips for each sample $24 \mathrm{~h}$ after printing. The adhesion was quantified by image analysis of the mark on the overprinted ink film, and the tape strip (stuck to a print) was compared to the mark on the ink film of the original Process ink and to the tape strip without the transferred ink. The adhesion was quantified on a fivestep scale: 1 -the appearance of the print does not change, 2-the appearance of the print does not change, there is no damage of the dried ink layer, only a slight changes of color, 3 - the appearance of the print does not change, in some places small loss of dried ink layer is visible, 4-the appearance of the print change, a significant part of dried ink layer is removed, and 5-the appearance of the print change, the dried ink layer is almost completely removed by tape.

A SpectroEye spectrophotometer (GretagMacbeth, Switzerland) was used to determine the optical densities of the full-tone area (i.e. the area was fully covered by the printing ink) and to determine the specific ink color components $L^{*}, a^{*}, b^{*}$ of dried ink layers. The optical density measurements for the full-tone area were performed with D50 illuminant using a $2^{\circ}$ observer, $0^{\circ} / 45^{\circ}$ measuring geometry and a polarization filter. The measurements of $L^{*}, a^{*}, b^{*}$ were carried out using these settings without a polarization filter, according to the ISO standard. ${ }^{27}$ The reported results are the average of the measurements from a minimum of six areas (optical densities), from which three color components $\left(L^{*}, a^{*}, b^{*}\right)$ were obtained on two different samples.

Colorfastness to dry and wet rubbing of the prints was studied one week after printing, using an Ink Rub Tester (TMI Machines, Canada), according to the TAPPI standard. ${ }^{28}$ An offset paper strip (90 g, Arctic Paper Kostrzyn, Poland) was attached to a weight $(0.9 \mathrm{~kg})$ and automatically rubbed 100 times at a speed of 100 cycles $\mathrm{min}^{-1}$ along the overprinted sample. For the wet-rub resistance measurements between the sample and the paper attached to a weight $(0.9 \mathrm{~kg})$, $0.1 \mathrm{~mL}$ of water (five drops of water) was put on the sample. The dry- and wet-rub resistance of the prints was evaluated by measuring the $L^{*}, a^{*}, b^{*}$ values of the sample after 30 rub cycles. Additionally, the dry-rub resistance was quantified by analyzing the color of the paper strip on a gray scale after 30, 50 and 100 cycles, providing that the sample was not damaged. The measurements were performed on two different overprinted samples.

The gloss of the films and prints was measured at 20, 60 and $85^{\circ}$ geometry conditions using a Micro-TriGloss gloss meter (BYK-Gardner, Germany). Data collection was performed at six different positions of the samples in both the cross and machine directions, and the reported values are the average of these measurements.

\section{Results and discussion}

\section{Polymer synthesis and characterization}

In this work polyglycerol, BPG, was synthesized through a ring-opening polymerization from glycidol using deprotonated 1-butanol as a starting material; thus, the BPG exhibited an asymmetric structure. Furthermore, obtained BPG act as a compatibilizer, wherein the hydrophobic butyl alkyl chain in a BPG macromolecule interacts with the surface of plastic base and the hydrophilic hydroxyl end groups interacts with the water-based ink. Our previous work shows that branched glycerols have a positive effect on the wettability, the adhesion, and wet or dry-rub resistance. ${ }^{29}$

Figure 1 shows the formation of BPG through an anionic ring-opening polymerization from glycidol. Deprotonated 1-butanol was used as an initiator for the anionic polymerization reaction. Four types of structural units may be formed in branched glycerol ${ }^{20,30,31}$ : linear $1,3\left(L_{1,3}\right)$, linear 1,4 $\left(L_{1,4}\right)$, dendritic $(D)$ and terminal 1,2 $\left(T_{1,2}\right)$ units. These structural units were determined with ${ }^{13} \mathrm{C}$ NMR spectra (see Fig. 2). The formation of linear, dendritic and terminal units is widely discussed in a previous publication. ${ }^{20}$ Secondary alkoxides are known to isomerize into primary alkoxides, and it is well known 


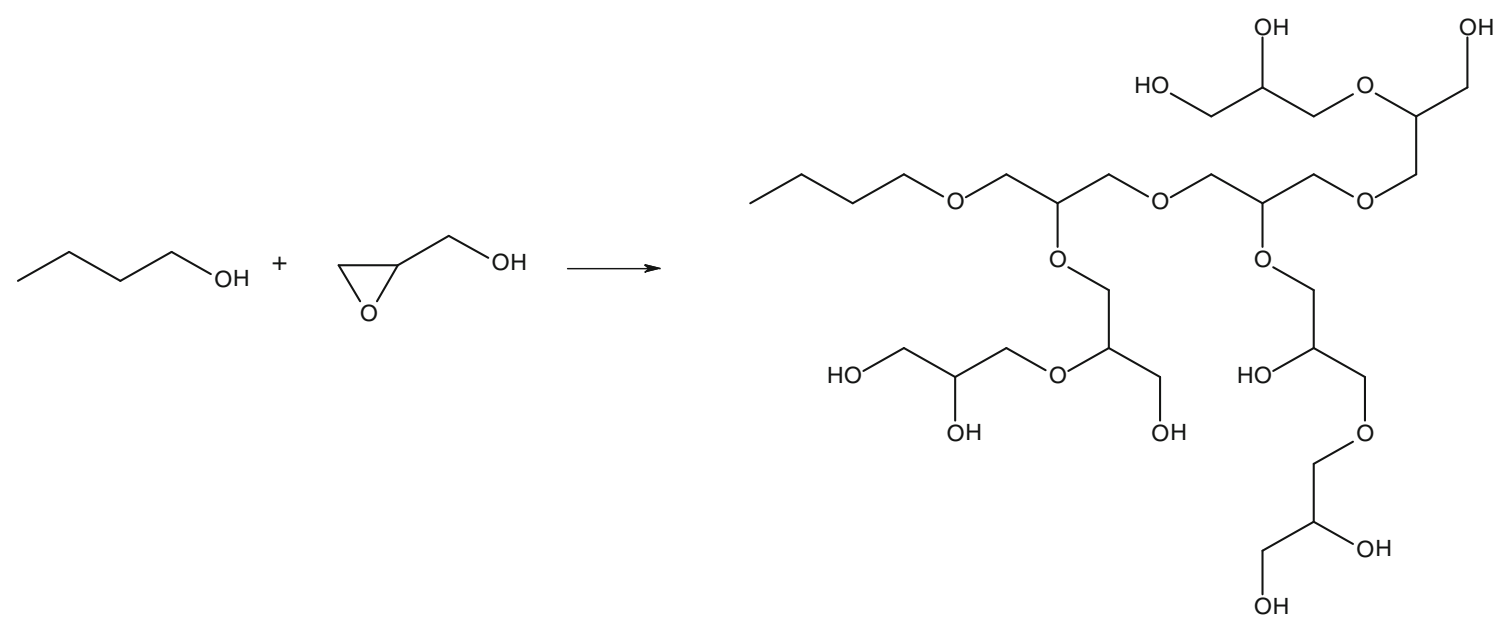

Fig. 1: Reaction scheme of anionic ring opening polymerization of glycidol

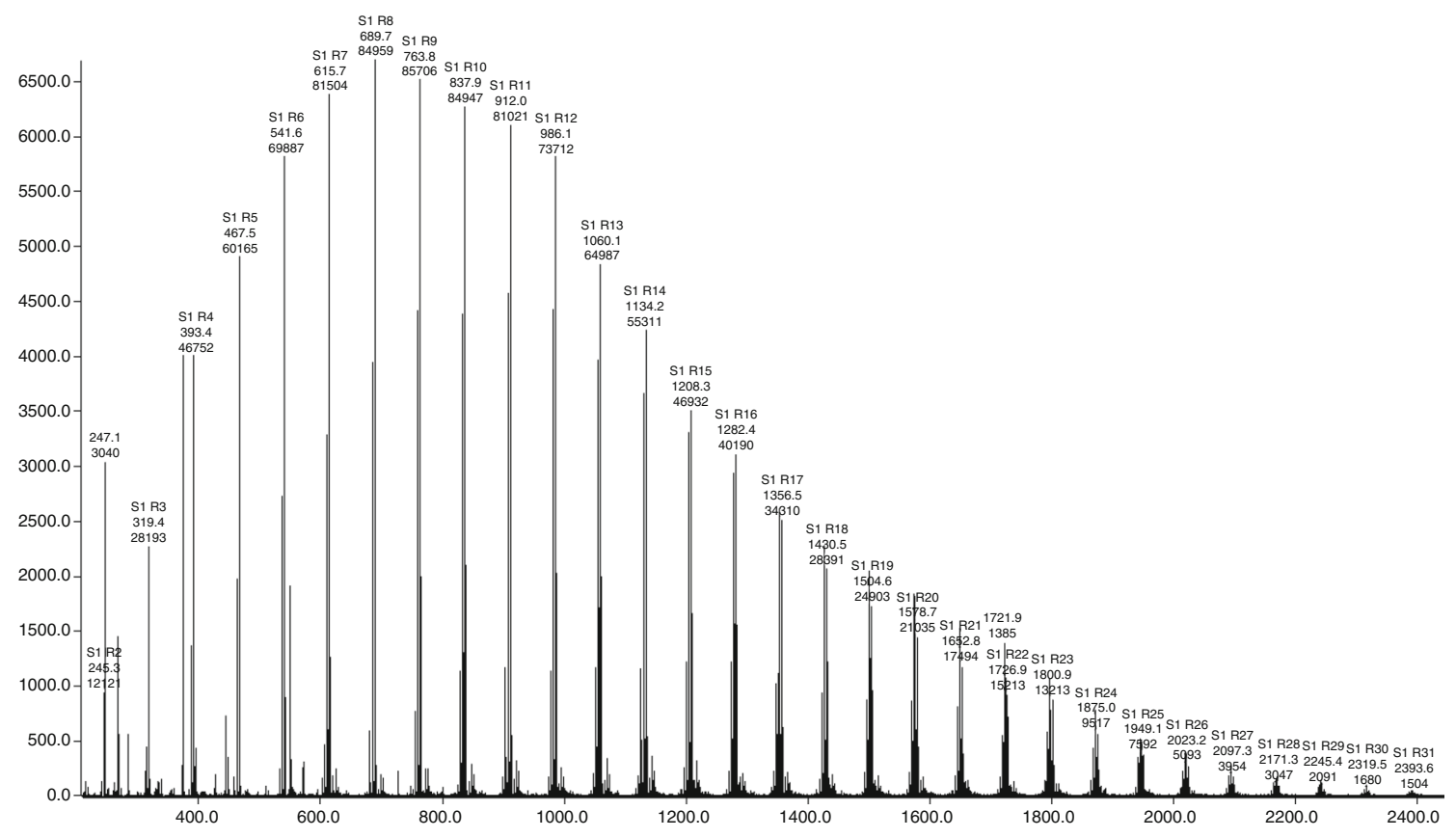

Fig. 2: MALDI-ToF mass spectrum of BPG

that secondary alkoxides are more stable, but less reactive, than primary alkoxides. If the primary alkoxide is propagated with glycidol, the $L_{1,4}$ unit is formed, and if the secondary alkoxide is propagated, the $L_{1,3}$ is created. The branched $D$ unit is generated when both hydroxyl groups have reacted with glycidol. The $T_{1,2}$ unit, with two hydroxyl groups, is formed by proton exchange or final acid addition. Additionally, in the ${ }^{13} \mathrm{C}$ NMR spectrum, the signals from the starting material are observed: 14.49 $\left(\mathrm{CH}_{3}\right), 19.52\left(\mathrm{CH}_{3}-\mathrm{CH}_{2}\right)$ and $13.97\left(\mathrm{CH}_{2}\right)$ ppm. The chemical shifts of the $L_{1,3}, L_{1,4}, D$ and $T_{1,2}$ units of BPG were determined based on previously published data. $^{20,22,30,32}$
The methyl and methylene groups of the starting material (1-butanol) appeared at 0.86, 1.24-1.36 and 1.38-1.49 ppm in the ${ }^{1} \mathrm{H}$ NMR spectrum. The protons from the methylene and methane groups of the polyglycerol occurred as a multiplet between 3.2 and $3.7 \mathrm{ppm}$. The hydroxyl groups appeared around 4.4$4.8 \mathrm{ppm}$. In the FTIR spectrum of BPG, an absorption band at $3374 \mathrm{~cm}^{-1}$, characteristic for hydroxyl groups, could be observed.

Figure 3 shows the MALDI-TOF mass spectrum of BPG. The weight-average molecular weight $\left(M_{\mathrm{w}}\right)$, number-average molecular weight $\left(M_{\mathrm{n}}\right)$ and polydispersity (PDI) of BPG, determined from the analysis of 


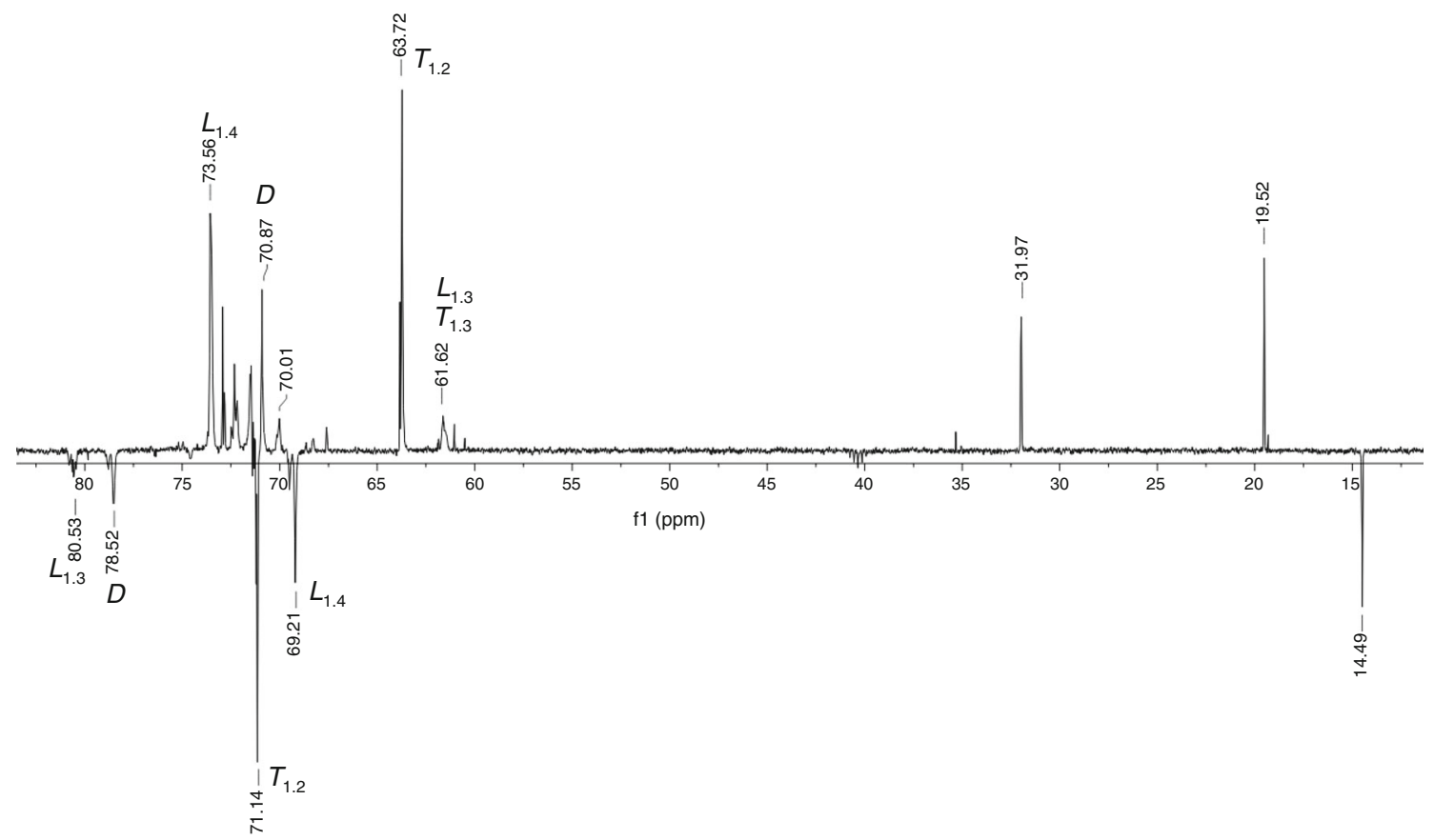

Fig. 3: ${ }^{13} \mathrm{C}$ NMR (400 MHz, DMSO- $d_{6}$ ) spectrum of BPG

Table 1: $M_{w}, M_{n}, P D I$ and the number of repeat units of $B P G$, according to mass spectrometry results

\begin{tabular}{cccc}
$M_{\mathrm{w}}$ & $M_{\mathrm{n}}$ & DPI & $\begin{array}{c}\text { Number of } \\
\text { repeat units }\end{array}$ \\
\hline 1203 & 942 & 1.3 & $15^{\mathrm{a}}$ \\
\hline
\end{tabular}

a Estimated from $M_{\mathrm{w}}$

the mass spectrum, are presented in Table 1 . The mass increment of the repeating unit is $74 \mathrm{Da}$, and corresponds to a fragment of glycerine. The BPG exhibited a low molecular weight, $M_{\mathrm{w}}=1200$. From the $M_{\mathrm{w}}$, the number of repeating units was estimated to be approximately 15 monomers and 16 hydroxyl groups for BPG. The polydispersity of BPG was quite low with a PDI of 1.3 .

\section{Print quality}

In this work, the print quality was determined as the properties of the print (i.e., the optical density of the full-tone area), the color values $\left(L^{*}, a^{*}, b^{*}\right)$ and gloss, as well as the mechanical properties of print (i.e. the adhesion of the dried ink film to the plastic film and the dry- and wet-rub resistance).

Before printing, the investigated inks (the original Process ink and the ink after the addition of 0.01 BPG) were tested using a flow cup. Flexographic printing requires ink flow times within 18-35 s (outlet diameter $4 \mathrm{~mm}$ ), according to a previously published report. ${ }^{1}$
The flow time of the original Process ink was $17.0 \mathrm{~s}$, and the printing ink modified with BPG was $17.1 \mathrm{~s}$; therefore, the addition of the oligomer had no impact on the flow time of the ink, which has also been observed by us previously. ${ }^{17,18}$

The adhesion of the ink film to the base (oPP or PET plastic films) should be examined before the optical density, color values $\left(L^{*}, a^{*}, b^{*}\right)$ and mechanical properties of the print are measured. It is necessary to assess the adhesion of the ink film to the base, because this parameter determines the print quality as well as the interactions between the overprinted ink film and the base. In industry, the adhesion of the ink film to the base is quantified using a test tape. ${ }^{26,33}$ The adhesion was quantified on a five-step scale; the results are presented in Table 2.

Adhesion of the original Process ink on an oPP film was generally much better than adhesion on a PET film. Unfortunately, for the ink modified with BPG, the adhesion of the dried ink film to the base was significantly worse.

The color values $\left(L^{*}, a^{*}, b^{*}\right)$, optical density and gloss values are presented in Table 3 . The total color differences $\left(\Delta E_{a b}{ }^{*}\right)$ were calculated from equation $(1)^{34}$ :

$\Delta E_{a b}^{*}=\sqrt{(\Delta L)^{2}+\left(\Delta a^{*}\right)^{2}+\left(\Delta b^{*}\right)^{2}}$

where $\Delta L^{*}=L_{\mathrm{BPG}}{ }^{*}-L^{*}, \Delta a^{*}=a_{\mathrm{BPG}}^{*}-a^{*}$ and $\Delta b^{*}=$ $b_{\mathrm{BPG}}^{*}-b^{*}$ are the differences between the value of the ink with added BPG and the original Process ink. The $L^{*}$ value is a measure of the lightness of an object, and 
is quantified on a scale where $L^{*}=0$ for a perfect black diffuser and $L^{*}=100$ for a perfect reflecting diffuser. The $a^{*}$ value is a measure of the redness (positive $a^{*}$ ) or greenness (negative $a^{*}$ ). The $b^{*}$ value is a measure of yellowness (positive $b^{*}$ ) or blueness (negative $b^{*}$ ). The addition of BPG increases the $L^{*}$, $a^{*}, b^{*}$ values of samples overprinted on oPP and PET films, whereas the $L^{*}, a^{*}, b^{*}$ values are twice as high for overprinted oPP films. The values change from $L^{*}=24.60, a^{*}=-0.42$ and $b^{*}=-1.50$ for the original Process ink to $L^{*}=32.85, a^{*}=1.38$ and $b^{*}=2.11$ for the ink with added BPG. The lowest value of $\Delta \mathrm{E}_{a b}$ * was observed for the overprinted PET $\left(\Delta E_{a b}{ }^{*}=4.0\right)$. The $\Delta E_{a b} *$ value indicates whether the color difference is acceptable and it can be observed by the human naked eye.

The optical density is a fundamental parameter that describes the quality of a print. The optical density assesses the thickness of the dried ink layer; higher values refer to thicker dried ink layers. It is well known that the thickness of the dried ink layer depends on the wettability of the base by the printing ink during the printing process: better wettability is connected with a thinner dried ink layer and lower optical density. In general, the optical density of full-tone area decreases with BPG addition. The optical density was higher for the overprinted oPP film with the original Process printing ink compared to that for the overprinted PET films. Moreover, the difference in the optical density between the original Process ink and the printing ink with added BPG was greater for the overprinted oPP film. It can be concluded that a small addition of hyperbranched oligoether changes the color properties

Table 2: Adhesion of the dried ink film to the basequality test

\begin{tabular}{lc} 
Sample & Adhesion \\
\hline oPP & 1 \\
Process & 3 \\
Process + BPG & 3 \\
PET & 4 \\
Process & 4 \\
Process + BPG & \\
\hline
\end{tabular}

of the overprinted ink film, which was also observed in previous studies. ${ }^{17,18}$

Gloss is one of the most important parameters in printing and packaging technology, because gloss affects the print quality by providing a better overall look and greater depth to colors. ${ }^{35,36}$ Gloss is a property of a material that is responsible for light reflection from a surface. The methods for measuring gloss depend on the kind of base that is used. The original Process printing ink is a middle-high gloss, or a semi-gloss, so the gloss values were in the range 3070 gloss units (GU). Hence, a measuring geometry with an angle of $60^{\circ}$ was used, according to the ISO standard. ${ }^{37}$ The addition of BPG to the Process printing ink increases the gloss of the prints. The differences are in the range of $7.7 \mathrm{GU}$ for oPP prints and $9.1 \mathrm{GU}$ for PET prints. The changes observed in the gloss value were greater in previous works, which studied solvent flexographic printing inks after the addition of 0.01 hyperbranched polyesters. ${ }^{17,18}$

\section{Colorfastness}

Table 4 presents the values of the $L^{*}, a^{*}, b^{*}$ after 30 cycles of dry- and wet-rub testing for the overprinted oPP film. The dried ink film on PET was damaged after 30 cycles, which prevented color measurements. The $\Delta E_{a b} *(30)$ value was calculated, according to equation (1), as a change between the values of the $L^{*}, a^{*}, b^{*}$ of the ink after 30 cycles of rubbing and before rubbing. The $\Delta E_{a b} *(30)$ value for dry rubbing was much lower for the ink with added BPG than for the original printing ink; $\Delta E_{a b} *(30)=0.3$ for the ink with added BPG and $\Delta E_{a b} *(30)=1.9$ for the Process ink. The differences probably occurred because BPG acts as a wax, which improves the colorfastness to rubbing. For printing inks, it is important for cohesion forces to occur between the molecules in the dried ink film, besides adhesion forces between dried ink layer and the base. ${ }^{38}$ The addition of BPG improved the dry rub resistance and did not improve the adhesion, so the cohesion forces in the dry ink layer are stronger than adhesion forces between the dry ink layer and the plastic film base, due the cross-hydrogen bonding of hydroxyl groups in the BPG molecule and the resin in printing ink. The wet-rub resistance of

Table 3: $L^{*}, a^{*}, b^{*}$ values, $\Delta E_{a b^{*}}$, optical densities and gloss of ink layers printed with the investigated printing inks

\begin{tabular}{|c|c|c|c|c|c|c|}
\hline Sample & $L^{*}$ & $a^{*}$ & $b^{\star}$ & $\Delta E_{a b^{*}}$ & $D$ & Gloss (GU) \\
\hline \multicolumn{7}{|l|}{ oPP } \\
\hline Process & 24.60 & -0.42 & -1.05 & 9.0 & 2.45 & 56.1 \\
\hline Process + BPG & 32.85 & 1.38 & 2.11 & & 1.66 & 63.8 \\
\hline \multicolumn{7}{|l|}{ PET } \\
\hline Process & 25.21 & 0.26 & 0.31 & 4.0 & 2.26 & 48.2 \\
\hline Process + BPG & 29.07 & 0.91 & 1.32 & & 1.84 & 57.3 \\
\hline
\end{tabular}


Table 4: Color values $L^{*}, a^{*}, b^{*}$ and $\Delta E_{a b^{*}}(30)$ for overprinted oPP films, measured after 30 rub cycles for dry and wet rubbing

Sample

Rub cycles

\begin{tabular}{|c|c|c|c|c|c|c|c|c|}
\hline & \multicolumn{4}{|c|}{ Dry } & \multicolumn{4}{|c|}{ Wet } \\
\hline & \multicolumn{4}{|c|}{30} & \multicolumn{4}{|c|}{30} \\
\hline & $L^{*}$ & $a^{*}$ & $b^{*}$ & $\Delta E_{a b}{ }^{\star}(30)$ & $L^{*}$ & $a^{*}$ & $b^{*}$ & $\Delta E_{a b}{ }^{*}(30)$ \\
\hline Process & 22.75 & -0.58 & -1.64 & 1.9 & 24.94 & 0.41 & -0.54 & 1.0 \\
\hline Process + BPG & 32.77 & 1.51 & 2.34 & 0.3 & 32.59 & 2.10 & 2.07 & 0.8 \\
\hline
\end{tabular}

Calculated as differences between the value of the ink after 30 cycles of rubbing and before rubbing

Table 5: Colorfastness to dry and wet rubbing, as a function of the number of rub cycles, for the investigated samples. The values are quantified on a gray scale (1-5)

Sample

Rub cycles

\begin{tabular}{llll}
\hline & Dry & & Wet \\
\hline 30 & 50 & 100 & 30 \\
\hline 2 & 3.5 & 4 & \\
1 & 1 & 1.5 & 2.5 \\
2.5 & 3.5 & 4 & 3 \\
1.5 & 2 & 3 & 2 \\
\hline
\end{tabular}

overprinted plastic films with water-based inks is usually poor. ${ }^{18}$ In this case, the Process printing ink is characterized by having a better colorfastness to wet rubbing $\left[\Delta E_{a b} *(30)=1.0\right]$ than to dry rubbing $\left[\Delta E_{a b} *(30)=1.9\right]$. The poorer wet-rub resistance of the printing ink containing BPG may, in general, be related to the high solubility of polyglycerols in water.

The values of colorfastness to dry and wet rubbing, according to a gray scale as a function of rub cycles are presented in Table 5. The color of the paper strip, quantified on a gray scale (1-5), increases with the number of rub cycles. The overprinted oPP films showed slightly better colorfastness to rubbing than the overprinted PET films, which was expected, because the adhesion of the printing ink to oPP was slightly better. However, the prints were characterized as having a much higher resistance to dry rubbing than to wet rubbing, which is typical for water-based printing inks. Also, better colorfastness to dry and wet rubbing was observed for the printing ink that contained BPG. The change in colorfastness to dry and wet rubbing is noticeable; for overprinted oPP films, it decreased from 2 for the Process ink to 1 for the ink with added BPG for dry rubbing, and from 2.5 for the Process ink to 2 for the ink with added BPG for wet rubbing. Generally, the addition of BPG to the Process ink improved the colorfastness to rubbing for ink films printed on both PET and oPP bases.

\section{Conclusions}

In this study, an asymmetric, hyperbranched oligoglycerol with a low molecular mass $\left(M_{\mathrm{w}}=1200\right)$ was presented as a performance additive for water-based printing ink. The synthesis of polyglycerol BPG was described and the oligomer was properly characterized; the structure of BPG was presented. The impact of BPG on the print quality, that is, the $L^{*}, a^{*}, b^{*}$ color values, optical density, full-tone area, and colorfastness to dry and wet rubbing, was reported. A comparison of the colorimetric parameters of plastic films overprinted with Process printing ink and ink containing BPG showed differences in the $L^{*}, a^{*}$, $b^{*}$ values. The overprinted samples that were produced with the printing ink containing BPG exhibited colorfastness to dry and wet rubbing that was superior to films of the original Process ink.

In conclusion, the investigated hyperbranched polyglycerol, BPG, can be used to modify water-based printing inks that are dedicated to printing on plastic films. Hyperbranched polyglycerols of low molecular mass have good potential to find applications in the print market.

Acknowledgments Funding for this research was provided by National Science Centre based on decision nr DEC-2013/11/D/ST8/03371. The authors thank Michael Huber (Poland) for the ink sample. 
Open Access This article is distributed under the terms of the Creative Commons Attribution License which permits any use, distribution, and reproduction in any medium, provided the original author(s) and the source are credited.

\section{References}

1. Havlínová, B, Cicák, V, Brezová, V, Horňáková, Ľ, “WaterReducible Flexographic Printing Inks-Rheological Behaviour and Interaction with Paper Substrates." J. Mater. Sci., 34 (9) 2081-2088 (1999)

2. Rentzhog, M, Fogden, A, "Print Quality and Resistance for Water-Based Flexography on Polymer-Coated Boards: Dependence on Ink Formulation and Substrate Pretreatment." Prog. Org. Coat., 57 (3) 183-194 (2006)

3. Seiler, M, "Hyperbranched Polymers: Phase Behavior and New Applications in the Field of Chemical Engineering." Fluid. Phase Equlib., 241 (1-2) 155-174 (2006)

4. Mallepally, RR, Smirnova, I, Arlt, W, Seiler, M, KleeLaquai, SK, Hills, G, "Enzymatic Degradation of Hyperbranched Polyesters." J. Appl. Polym. Sci., 112 (4) 18731881 (2009)

5. Gao, C, Xu, Y, Yan, D, Chen, W, "Water-Soluble Degradable Hyperbranched Polyesters: Novel Candidates for Drug Delivery ?" Biomacromolecules, 4 704-712 (2003)

6. Inoue, K, "Functional Dendrimers, Hyperbranched and Star Polymers." Prog. Polym. Sci., 25 (4) 453-571 (2000)

7. Žagar, E, Žigon, M, “Aliphatic Hyperbranched Polyesters Based on 2,2-Bis(methylol)propionic Acid-Determination of Structure, Solution and Bulk Properties." Prog. Polym. Sci., 36 (1) 53-58 (2011)

8. Jikei, M, Kakimoto, M, "Hyperbranched Polymers: A Promising New Class of Materials." Prog. Polym. Sci., 26 (8) 1233-1285 (2001)

9. Tryznowski, M, Tomczyk, K, Fraś, Z, Gregorowicz, J, Rokicki, G, Wawrzyńska, W, Parzuchowski, P, “Aliphatic Hyperbranched Polycarbonates: Synthesis, Characterization, and Solubility in Supercritical Carbon Dioxide." Macromolecules, 45 (17) 6819-6829 (2012)

10. Daniel, W, Stiriba, SE, Holger, F, "Hyperbranched Polyglycerols: From the Controlled Synthesis of Biocompatible Polyether Polyols to Multipurpose Applications." Acc. Chem. Res., 43 (1) 129-141 (2010)

11. Frey, H, Haag, R, "Dendritic Polyglycerol: A New Versatile Biocompatible Material." Rev. Mol. Biotechnol., 90 (3-4) 257-267 (2002)

12. Calderón, M, Quadir, MA, Sharma, SK, Haag, R, “Dendritic Polyglycerols for Biomedical Applications." Adv. Mater., 22 (2) 190-218 (2010)

13. Moore, E, Thissen, H, Voelcker, NH, "Hyperbranched Polyglycerols at the Biointerface." Prog. Surf. Sci., 88 (3) 213-236 (2013)

14. Ekinci, D, Sisson, A, Lendlein, A, "Polyglycerol-Based Polymer Network Films for Potential Biomedical Applications." J. Mater. Chem., 22 (39) 21100-21109 (2012)

15. Maessen, P, Van, AH, "A Method for Treating a Lithographic Printing Plate." EP Patent 2,002,987 A1, 2008

16. Hayashi, A, Ishimaru, Y, "Inkjet Aqueous Ink and Printed Item." US Patent 7,955,427 B2, 2010

17. Żołek-Tryznowska, Z, Izdebska, J, "Hyperbranched Polymers-Their Application in Printing Inks." Compos. Interfaces, 19 (7) 441-451 (2012)
18. Żołek-Tryznowska, Z, Izdebska, J, "Flexographic Printing Ink Modified with Hyperbranched Polymers: Boltorn ${ }^{\mathrm{TM}}$ P500 and Boltorn ${ }^{\text {TM }}$ P1000." Dyes Pig., 96 (2) 602-608 (2013)

19. McKee, MG, Unal, S, Wilkes, GL, Long, TE, "Branched Polyesters: Recent Advances in Synthesis and Performance." Prog. Polym. Sci., 30 (5) 507-539 (2005)

20. Sunder, A, Hanselmann, R, Frey, H, Mülhaupt, R, "Controlled Synthesis of Hyperbranched Polyglycerols by RingOpening Multibranching Polymerization." Macromolecules, 32 (13) 4240-4246 (1999)

21. Paulus, F, Weiss, MER, Steinhilber, D, Nikitin, A, Schütte, C, Haag, R, "Anionic Ring-Opening Polymerization Simulations for Hyperbranched Polyglycerols with Defined Molecular Weights." Macromolecules, 46 (21) 8458-8466 (2013)

22. Tokar, R, Kubisa, P, Penczek, S, Dworak, A, "Cationic Polymerization of Glycidol: Coexistence of the Activated Monomer and Active Chain End Mechanism." Macromolecules, 27 (2) 320-322 (1994)

23. Sunder, A, Bauer, T, Mülhaupt, R, Frey, H, "Synthesis and Thermal Behavior of Esterified Aliphatic Hyperbranched Polyether Polyols." Macromolecules, 33 (4) 1330-1337 (2000)

24. ISO 2431:2011, Paints and Varnishes-Determination of Flow Time by Use of Flow Cups

25. ISO 3270:1984, Paints and Varnishes and Their Raw Materials-Temperatures and Humidities for Conditioning and Testing

26. BS EN 15386:2007, Packaging. Flexible Laminate and Plastic Tubes. Test Method to Determine the Print Adhesion

27. ISO 13655:2009, Graphic Technology—Spectral Measurement and Colorimetric Computation for Graphic Arts Images

28. TAPPI T830, Ink Rub Test of Containerboard, (2004)

29. Żołek-Tryznowska, Z, Izdebska, J, Tryznowski, M, "Branched Polyglycerols as Performance Additives for Water-Based Flexographic Printing Inks." Prog. Org. Coat., (2014). doi:10.1016/j.porgcoat.2014.07.015

30. Rokicki, G, Rakoczy, P, Parzuchowski, P, Sobiecki, M, "Hyperbranched Aliphatic Polyethers Obtained from Environmentally Benign Monomer: Glycerol Carbonate." Green Chem., 2005 (7) 529-539 (2005)

31. Cao, L-C, Mou, M, Wang, Y, "Hyperbranched and viologenfunctionalized polyglycerols: Preparation, Photo- and Electrochromic Performance." J. Mater. Chem., 2009 (19) 34123418 (2009)

32. Cassel, S, Debaig, C, Benvegnu, T, Chaimbault, P, Lafosse, M, Plusquellec, D, Rollin, P, "Original Synthesis of Linear, Branched and Cyclic Oligoglycerol Standards." Eur. J. Org. Chem., 2001 (5) 875-896 (2001)

33. Eldred, NL, What the Printer Should Know About Ink, 3rd ed. Graphic Arts Technical Foundation Press, Pittsburgh, PA (2001)

34. ISO 7724-3:1984, Paints and Varnishes-Colorimetry-Part 3: Calculation of Colour Differences

35. Jančovičová, V, Kindernay, J, Jakubíková, Z, Mrlláková, I, "Interactions in Iron Gall Inks." Chem. Pap., 61 (5) 383-390 (2007)

36. Jančovičová, V, Mikula, M, Havlínová, B, Jakubíková, Z, "Influence of UV-Curing Conditions on Polymerization Kinetics and Gloss of Urethane Acrylate Coatings." Prog. Org. Coat., 76 (2-3) 432-438 (2013)

37. ISO 2813:1994, Paints and Varnishes-Determination of Specular Gloss of Non-metallic Paint Films at 20 Degrees, 60 Degrees and 85 Degrees

38. Żołek-Tryznowska, Z, Izdebska, J, Gołąbek, M, "Ionic Liquids as Performance Additives for Water-Based Printing Inks." Color. Technol., 130 (4) 314-318 (2014) 